\title{
PSICOPATOLOGÍA Y REALIDAD VIRTUAL
}

\author{
ROSA M. BAÑOS ${ }^{1}$, CRISTINA BOTELLA ${ }^{2}$ y CONCHA PERPIÑA ${ }^{1}$ \\ ${ }^{1}$ Universidad de Valencia \\ ${ }^{2}$ Universidad Jaime I de Castellón
}

(Recibido el 11 de septiembre de 1998)

\begin{abstract}
La Realidad Virtual (RV) se está empezando a considerar como una importante herramienta para la investigación en muchos y muy variados campos. Actualmente, los progresos en el desarrollo de esta tecnología están yendo incluso más rápido de lo que podemos anticipar, por lo que reflexionar sobre sus potenciales usos y abusos resulta algo totalmente necesario. Este trabajo se centra en analizar algunas de las aplicaciones e implicaciones que la RV puede tener para la Psicopatología. Obviamente, éstas pueden ser muy diversas, pero aquí nos centramos sólo en tres: a) La RV como un contexto de laboratorio "realista" donde estudiar comportamientos, emociones, pensamientos, etc., problemáticos; b) la RV como un modelo para abordar algunas preguntas centrales, que desde siempre han intrigado a los psicopatólogos; y c) la RV y posibles experiencias ¿psicopatológicas?. Creemos que la RV se puede constituir en una herramienta que permita a la Psicopatología retar y modificar posiciones teóricas establecidas, a la par que plantearse otras nuevas. Esta nueva forma de experiencia, a la vez que puede ayudarnos a encontrar respuestas a viejas preguntas, puede también hacer surgir otras nuevas en el horizonte psicopatológico y psicológico, y no sólo relacionadas con cuestiones teóricas o empíricas, sino también morales. Este camino de investigación no ha hecho más que empezar.
\end{abstract}

Palabras clave: Realidad virtual, psicopatología, juicio de realidad.

\section{Psychopathology and virtual reality}

Virtual Reality (VR) is becoming an important tool in the research on many and various fields. Today, the progresses in the development of this technology are going even faster than we expected, and therefore it is being necessary to think over its potential uses and abuses. This paper focuses in the analysis of some of the applications and implications of VR on Psychopathology. Although we are plenty aware of the broad range capable of being covered, we have focused on: a) VR as a "realistic" lab setting where problematic behaviours, affects, thoughts, etc. could be studied; b) VR as a model to deal with certain core questions which have always been of interest for psychopathologists; and c) VR as experiences open to be labelled as psychopathological. We believe that VR has the potential of becoming the tool that will enable Psychopathology to challenge and to modify theoretical assumptions already established, as well as to help us to find new answers to old questions. VR could also make new questions rise on the psychological and psychopathological horizons, questions not only related to theoretical or empirical issues, but also to moral ones. This line of research has just started.

Key words: Virtual reality, psychopathology, reality judgement.

\section{INTRODUCCIÓN}

Que la Realidad Virtual (RV) será una
herramienta muy importante para la
investigación en muchos y muy variados

Agradecimientos: La realización de este trabajo ha sido posible, en parte, gracias a la subvención de los proyectos de investigación concedidos por la Generalitat Valenciana (GV-D-ES-17-123-96) y por la Generalitat Valenciana (IMPIVA) (971801003556). campos creemos que es algo difícil de poner en duda. Actualmente, los progresos en el desarrollo de esta tecnología están yendo incluso más rápido de lo que podemos anticipar (Tart, 1991). Sin

Correspondencia: Rosa María Baños. Facultad de Psicología. Universidad de Valencia. Dpto. Personalidad, Evaluación y Tratamientos Psicológicos. Avda. Blasco Ibáñez, 21, 46010- Valencia. Tfno.: 963864476. Correo-e: banos@uv.es. 
embargo, lo que parece menos claro es que hayamos topado con una herramienta que vaya a poder respondernos a cualquier pregunta que nos planteemos, sea cual sea el campo de interés. Es por eso que creemos necesario que cada área de estudio, y en este caso las áreas de estudio psicológicas, reflexionen sobre cuáles son los usos (y los abusos) que se pueden hacer de esta nueva tecnología. Este trabajo se centra precisamente en analizar algunas de las aplicaciones e implicaciones que la RV puede tener para la Psicopatología. En una disciplina como ésta parece fácil, al menos a simple vista, pensar en múltiples posibilidades de la RV. Sin embargo, muchas de ellas serían hoy por hoy ciencia-ficción, lo cual no quiere decir que en un futuro no muy lejano no se puedan realizar, ya que los "futuros» en tecnología parecen estar cada vez más cerca del presente, y últimamente nos estamos acostumbrando a que, además, superen nuestras propias ficciones. Sin embargo, no desearíamos que esta reflexión se volviese excesivamente especulativa, aunque no vamos a poder evitar que haya algo (o mucho) de especulación. En este sentido, e intentando tener lo más posible los pies en la tierra, o mejor dicho, en el ambiente virtual, nos centraremos en tres posibles aplicaciones:

1. La RV como un contexto de investigación para la Psicopatología, es decir, como un laboratorio psicológico-psicopatológico.

2. La RV como generadora de modelos útiles para estudiar procesos básicos y sus alteraciones.

3. Las posibles experiencias psicopatológicas, infrecuentes o anómalas que se pueden producir en RV.

\section{LA RV COMO LABORATORIO PSICOLÓGICO-PSICOPATOLÓGICO}

La RV puede concebirse como un contexto de laboratorio en el que estudiar comportamientos, emociones, pensamientos, etc., problemáticos. Y, en este sentido, creemos que este «laboratorio realista» podría ayudar a mejorar ciertos aspectos metodológicos que suelen debilitar algunas de las investigaciones actuales en Psicopatología. ¿Qué es lo que aportaría este «laboratorio»?

En Psicopatología, al igual que en otras disciplinas psicológicas, si queremos saber qué es lo que le ocurre a una persona con un problema determinado $\mathrm{y}$, además, aventurar algunas posibles explicaciones para ese problema, desde luego el camino más «directo» es preguntarle a la persona qué le pasa. Es decir, podríamos optar por obtener datos de las personas que presentan un problema psicológico, acudiendo a los diversos formatos de auto-informes verbales. Estos pueden ser muy diversos, y pueden ir desde pedir al sujeto que rememore la última vez que se le presentó el problema, y que intente describirnos lo más fielmente posible cómo se sentía, qué pensaba, etc.; o también podemos, por ejemplo, dar al sujeto una larga serie de auto-registros y pedirle que cuando le suceda intente responder a una serie de preguntas. La RV no sustituye en absoluto este modo de evaluar problemas, pero amplía el uso de esta estrategia, ya que además de que en nuestra consulta le preguntemos a la persona por lo que le pasa, podemos recrear un ambiente en el que lo observemos directamente. Desde luego, esto no se puede hacer con todos los problemas psicológicos, pero algunos sí son susceptibles de este análisis. El caso más claro es el de las fobias. Podemos situar al paciente en el espacio abierto que teme, o en la multitud que evita, y que nos narre qué piensa, qué siente, etc. $O$ podremos situarle en una reunión social y observar cómo interactúa con los demás. Además de facilitar la observación de la conducta del sujeto en ese momento, podríamos también obtener información no sólo del 
contenido de sus pensamientos sino de los procesos y la organización de éste. $Y$ todo ello sin salir de nuestro laboratorio o de nuestra consulta y pudiendo controlar un enorme número de variables, difícilmente controlables o modificables en los contextos naturales.

Ya hay incluso quien plantea (Tart, 1990) que los futuros tests de diagnóstico consistirán en que el cliente entre en diversas realidades virtuales estandarizadas, que sean representativas de la vida cotidiana, para evaluar las reacciones del individuo. Sería algo así como tener un surtido de escenas de trabajo, de hogar, de riñas de pareja, etc. Aunque muy sugerente, esta tecnología no parece que pueda estar disponible en un futuro inminente. Además, cuenta con el inconveniente de que tendríamos que simular los diferentes ambientes y/o personas significativas relevantes para cada individuo particular. Sin embargo, los avances espectaculares que se están realizando en este campo nos permiten augurar que no está excesivamente lejano el día en que la tecnología permita elaborar escenarios de este tipo.

En cualquier caso, la KV sí que permite ya estudiar la conducta de las personas en algunas situaciones cotidianas. Por ejemplo, jugar a determinados juegos de RV puede proporcionar ejemplos de los modos en que las personas actúan en escenarios naturales y no de laboratorio. Esta clase de estudio nos permite observar la conducta en un contexto, así como analizar las interacciones entre contexto y conducta. Como Calvert y Tan (1994) señalan, los juegos de RV tienen un gran potencial para explorar el papel que las situaciones tienen en la iniciación, mantenimiento, terminación y limitación de las conductas humanas. Por ejemplo, los mismos Calvert y Tan utilizan un juego de RV para estudiar la activación fisiológica y los pensamientos agresivos en niños y adolescentes cuando juegan un juego agresivo en RV.
Desde luego, esta estrategia también tiene limitaciones, tanto prácticas como éticas. Por ejemplo, Tart (1990) plantea como posibilidad para evaluar la paranoia crear un ambiente virtual que comience con una simulación "normal» de una habitación con una serie de personas y que, progresivamente, se vaya modificando para incluir movimientos ambiguos, sombras, expresiones faciales de las personas virtuales y otros elementos que hagan el ambiente más amenazador. Desde luego, estamos de acuerdo con Begelman (1991) en que este tipo de aplicaciones producen, cuanto menos, aprensión, ya que la RV paranoide planteada por Tart parece menos pensada para reflejar o evaluar paranoia que para inducirla. Así, personas normales podrían incluso empezar a mostrarse recelosas y su efecto sobre pacientes paranoides podría ser incluso el de potenciar el problema.

Al margen de estas extralimitaciones, pensamos que si estamos interesados en conocer qué es lo que le ocurre a una persona con un problema determinado, y queremos aventurar algunas posibles explicaciones para tal problema, la RV puede superar algunas de las limitaciones de los auto-informes y de la observación directa, herramientas imprescindibles, hoy por hoy, en la evaluación e investigación psicopatológica.

Por otro lado, otra estrategia muy utilizada en Psicopatología es el uso de análogos experimentales. Aquí se encontrarían, por ejemplo, las manipulaciones de estados emocionales realizadas en el laboratorio, que intentan ser análogos de aquellos que ocurren en la realidad. Así, si queremos saber qué ocurre cuando una persona está triste (p.ej., cómo funcionan sus procesos de memoria, o cómo codifica el ambiente, etc.) se puede inducir en el laboratorio un estado de ánimo triste. Esto se suele hacer mediante imaginación, visionado de películas, frases autoreferentes, hipnosis, etc. Y, a partir de 
ahora, esto también se podrá hacer mediante RV. En un laboratorio de RV se podría recrear un escenario "emocionalmente cargado", con el que el individuo pudiera interactuar, lo que supondría ciertas ventajas: por ejemplo, no tendría el problema de generar a la vez un estado alterado de conciencia, como ocurre con la hipnosis, o no tendría el problema de que el estado de ánimo fuera excesivamente efímero, como ocurre con la lectura de frases auto-referentes, ya que el «escenario emocionalmente cargado» no sólo serviría para inducir el estado de ánimo, sino que también podría servir para mantenerlo.

Estas inducciones pueden no sólo referirse a estados emocionales. Por ejemplo, antes comentábamos la crítica de Begelman a la evaluación de la paranoia propuesta por Tart, ya que podía constituirse en una inducción en personas normales de estados similares, en algunos aspectos, a los paranoides. En una línea similar estarían los trabajos sobre delirios y creencias anómalas que intentan estudiar si los delirios surgen como explicaciones normales de experiencias inusuales (p.ej. Maher, 1988a y b; Maher y Spitzer, 1992). Con este propósito, se han estudiado las explicaciones que dan sujetos normales a conductas no voluntarias que realizan y que han sido sugeridas en sesiones previas de hipnosis. Del mismo modo, podríamos estudiar qué tipo de explicaciones dan los sujetos a ambientes virtuales en los que ocurren acontecimientos poco usuales, como por ejemplo, y siguiendo con el ejemplo de Tart, introducir de vez en cuando un elemento en la periferia de la visión del individuo, pero que éste nunca pueda contemplarlo claramente.

Otra estrategia muy utilizada actualmente en Psicopatología consiste en la evaluación en el laboratorio de la ejecución de los pacientes en tareas que estudian procesos cognitivos básicos, como la atención, percepción, memoria, etc. Así, desde la perspectiva de la Psicopatología Cognitiva Experimental se suelen utilizar tareas experimentales, como el Stroop emocional, o la detección de puntos, o tareas de codificación, etc., que intentan mejorar al máximo posible las cuestiones de la validez interna. Es decir, estas tareas experimentales intentan resolver un problema central para la Psicopatología (al igual que para la mayoría de las disciplinas relacionadas con la Psicología) el control de las variables. Sin embargo, al intentar controlar al máximo posible las variables extrañas, se suelen utilizar estímulos que se presentan descontextualizados, normalmente palabras aisladas, por lo que carecen de significado completo, lo que hace que nos enfrentemos con problemas de validez ecológica. Es decir, el coste de aumentar la validez interna de este modo, muchas veces repercute en la validez externa del experimento. Por ejemplo, para probar la hipótesis de que los procesos atencionales juegan un papel importante en los trastornos de ansiedad, un experimento puede presentar dos tipos de estímulos (p.ej., palabras emocionalmente neutras y palabras amenazadoras para el individuo, que representen su objeto temido). Si los pacientes ansiosos detectan antes los estímulos amenazadores que los neutros, decimos que la atención juega un papel en la ansiedad. Sin embargo, ¿podemos saber a ciencia cierta a qué parte del ambiente real y cotidiano atenderá un paciente ansioso, a partir de su ejecución en una tarea de tiempo de reacción en la que le hemos presentado palabras aisladas? $\mathrm{O}$, por poner otro ejemplo, ¿realmente podemos saber cómo influirá el auto-esquema de un depresivo en la codificación de una situación continua y dinámica, sabiendo cómo responde en la ejecución de palabras auto-referentes aisladas?

Dejando a un lado la cuestión de que las palabras pueden tener significados diferentes en función de la frase en la que 
se encuentran, sigue estando la cuestión de que la palabra, el lenguaje, sólo representa al objeto, pero no es el objeto, o en términos de Korzybski, es el mapa pero no es el territorio. Desde luego, si queremos saber cómo funcionan ciertos procesos cognitivos, por ejemplo, cuando un individuo fóbico a los aviones está volando en uno, en vez de presentarle palabras como avión, altura, azafata, etc., sería mucho más ecológico evaluar su ejecución atencional dentro de un avión. Obviamente, nos encontraríamos con muchas dificultades si pretendiéramos introducir a un individuo en un avión de verdad, con un aparato que registrara sus movimientos oculares, otro que registrara su actividad fisiológica, etc., a la vez que también volamos nosotros a su lado preguntándole continuamente por su unidad subjetiva de ansiedad. Probablemente, hoy por hoy sería difícil entrar así en un avión y, es más, posiblemente pocos individuos fóbicos se ofrecerían a participar en tal experiencia. Sin embargo, esto sí lo podemos hacer en un laboratorio de RV. Imagínese por un momento la cantidad de variables que podríamos medir y de un modo bastante controlado. Por ejemplo, existe ya en el mercado un aparato que se puede instalar en el casco de la RV y que registra en cada momento y de un modo bastante fiable qué es lo que mira el individuo (qué punto exacto del ambiente) y durante cuánto tiempo, por lo que tendríamos una medida precisa de qué es lo que está atrapando la atención del sujeto, o a qué es lo que está evitando atender, con las consiguientes implicaciones para las teorías cognitivas sobre la relación entre atención y trastornos de ansiedad.

Hemos hablado de validez externa y de validez interna, es decir de validez «inferencial», sin embargo, si investigamos en RV hay otro aspecto de la validez que deberíamos tener en cuenta, se trata de la validez «fenomenológica». Brickman (1978) denomina así a los componentes que determinan si una experiencia se vive o no como real, es decir, si el experimento tiene o no significado para el sujeto experimental. Este tipo de validez puede contribuir a la validez inferencial del experimento. Así, asegurar una alta validez fenomenológica a veces puede servir para verificar la eficacia de la manipulación, es decir, si los sujetos creen que la manipulación fue real, y, a la vez, saber si se puede generalizar a otras situaciones similares. Sin embargo, la validez fenomenológica y la inferencial no están necesariamente relacionadas. Este tipo de validez es bastante paralelo al análisis del realismo de un experimento que ofrecían Aronson y Carlsmith en 1968. Estos autores desdoblaban el realismo en dos componentes: el realismo del experimento (en qué medida se implican vívidamente los sujetos en los procedimientos del experimento) y el realismo mundano (en qué medida son representativos los procedimientos de la vida cotidiana). La validez fenomenológica de la que habla Brickman podría contribuir a ambos tipos de realismo, pero es un concepto más amplio. Como antes señalábamos, significa si el experimento tiene o no significado para el sujeto experimental, es decir si lo experimenta» como real y si sus sentimientos, su conducta y sus consecuencias están referidos a un sistema más amplio de los valores del sujeto. La cuestión de la validez fenomenológica es central en la investigación en RV, ya que es importante que el individuo experimente la situación virtual como real, lo cual significa que tiene validez para él.

Según Brickman, los estudios que enfatizan la validez inferencial se denominan experimentos, mientras que los estudios que tipifican la fenomenológica son los juegos. A estos últimos nosotras añadiríamos también la RV. Tanto los juegos como la RV son ambientes relativamente seguros en los que las personas pueden tener acce- 
so a experiencias que, de otro modo, serían inaccesibles para ellos y, además, podemos observar cómo manejan esa experiencia. Tanto en uno como en otro debemos buscar una elevada validez fenomenológica si queremos que nuestro estudio tenga éxito. Es decir, la validez fenomenológica se vuelve un requisito importante para la RV y, a su vez, esta tecnología pone sobre el tapete la importancia de este tipo de validez a la hora de investigar en Psicología y en Psicopatología.

Como conclusión de este primer apartado, nos gustaría resaltar que, aunque somos conscientes de que hemos pecado de una simplicidad excesiva, creemos que los ejemplos propuestos dejan claro que la RV puede constituirse en un contexto de laboratorio válido y útil, experimentalmente hablando. No estamos diciendo que sea la mejor estrategia para investigar en Psicopatología, ni tan siquiera que pueda sustituir a otras que utilizamos actualmente. Sólo que nos ofrece un modo que, sabiéndolo utilizar correctamente, puede superar algunas de las limitaciones de las estrategias que ya utilizamos y, por tanto, puede ayudarnos a progresar en el camino de la comprensión de los fenómenos psicopatológicos.

Sin embargo, en Psicopatología, como en cualquier disciplina científica, tanto o más importante que los medios para responder preguntas (es decir, las estrategias que usamos para investigar) es el saber formular las propias preguntas. Y esto nos lleva directamente a la segunda aplicación que la RV puede tener para la Psicopatología.

\section{LA RV COMO GENERADORA DE MODELOS ÚTILES PARA ESTUDIAR PROCESOS BÁSICOS Y SUS ALTERACIONES}

Como hemos señalado en otro lugar (Perpiñá, Botella y Baños, 1997), la RV tiene un uso potencial para estimular el conocimiento científico, ya que esta tecnología puede servir como modelo de comprensión del mundo, del mismo modo que en su momento sirvió el teléfono o el computador. Y si una analogía o un modelo puede ser útil para describir cómo funciona la mente humana, también puede servir para comprender $\mathrm{y}$ definir sus anomalías. En el caso de la Psicopatología esto, de hecho, ya se está produciendo. Por ejemplo, Tart (1990, 1991) hace una propuesta muy sugerente, aunque un tanto pretenciosa, que le permite diferenciar incluso entre normalidad, neurosis y psicosis. Tart propone una analogía de la realidad física con la realidad virtual y plantea que ya vivimos en una variedad de realidades virtuales internamente generadas. Según este autor, cada uno de nosotros vive «dentro» de una máquina de simulación del mundo, puesto que no debemos olvidar que nuestras «percepciones» no son más que simulaciones, no son la misma realidad. Nosotros sólo somos conscientes de un patrón final de acontecimientos neuronales. Esta idea no es en absoluto novedosa, Helmoltz ya la señalaba hace más de un siglo y, actualmente, las teorías psicológicas consideran la percepción como un proceso constructivo mediante el que se interpretan los datos sensoriales. Lo único novedoso de la propuesta de Tart es que utiliza una analogía con la RV y, además, la aplica al estudio de la anormalidad.

Tart denomina a los acontecimientos neuronales de los que somos conscientes, junto con los otros que llevan a este patrón final, «Proceso de Simulación del Mundo», un mecanismo que crea la RV en la que vivimos experimentalmente. Según él, la estructura de nuestro Sistema Nervioso constituye nuestros auriculares estéreos, nuestras gafas, nuestro guante, etc. Es decir, considera que nuestra conciencia es un mecanismo de realidad vir- 
tual, una simulación del mundo. La función básica del Proceso de Simulación del Mundo es crear, mantener, expandir y actualizar modelos internos del mundo real, los cuales nos capacitan para sobrevivir y funcionar eficientemente en el mundo real. Si nuestro Proceso de Simulación del Mundo nos ofrece realidades virtuales que difieren del mundo real de algún modo significativo, comenzamos a comportarnos desadaptativamente, creando consecuencias en el mundo real y/o sufrimiento psicológico en nosotros mismos o los demás. Es decir, para Tart las desviaciones psicopatológicas se deben a Procesos de Simulación del Mundo inusuales. Tart aclara que la RV que constituye nuestra percepción del mundo es una clase particular de RV, una realidad que es ampliamente compartida en sus perfiles más amplios por otros miembros de una cultura concreta. Más concretamente, en su trabajo de 1990, Tart se pregunta ¿qué es una persona "normal»?: Alguien que aparentemente sólo tiene un patrón bien desarrollado que puede hacerse cargo de su Proceso de Simulación del Mundo. Es decir, que la personalidad cotidiana, unitaria, y que implícitamente controla el Proceso de Simulación del Mundo, produce una RV y una acción consecuente que entra dentro del rango normal. Por su parte, una persona «neurótica» es alguien cuyo Proceso de Simulación del Mundo difiere significativamente del de los normales, de tal modo que algunas áreas de la vida en la que los normales funcionan bien se vuelven áreas de sufrimiento y funcionamiento desadaptativo. Por último, una persona psicótica para Tart sería alguien que vive en una RV tan obviamente diferente del rango de la RV en la que viven los «normales», que se considera así: obviamente diferente. Siguiendo con esta misma analogía, Tart se mete aún más en la arena e incluso llega a definir algún trastorno específico. Concretamente habla del tras- torno de personalidad múltiple que, según este modelo, se caracterizaría porque la persona tiene dos o más patrones, o constelaciones de patrones, bien desarrollados que controlan el Proceso de Simulación del Mundo, de tal modo que la persona vive temporalmente en una RV que constituye una identidad, una personalidad, un estado de conciencia. Creemos que esta idea es bastante sugerente, aunque dudamos que el DSM o la CIE la introduzcan como criterio clasificador en sus próximas ediciones.

Por otro lado, además de ayudarnos a definir lo patológico, la investigación en RV también puede ayudarnos ofreciendo modelos para abordar el estudio de preguntas más particulares, pero igualmente centrales en Psicopatología. Por ejemplo, la RV puede ser una herramienta importante para estudiar los procesos implicados en el juicio de realidad (Perpiñá, Botella y Baños, 1997). Y, precisamente, ésta es una de las preguntas que más intriga y ha intrigado a las psicopatólogos. La respuesta a esta pregunta puede desentrañar alguna de las claves más importantes sobre la distinción entre la psicosis y la neurosis o, dicho de una manera más tradicional, entre la «locura» y la «normalidad».

Investigar sobre cómo puede influir la $\mathrm{RV}$ en los juicios de realidad que realizan las personas, quizá nos dé alguna luz para descubrir cómo adscribimos realidad a nuestras percepciones, cogniciones, interacciones, etc. y, especialmente, en qué fallan esos procesos metacognitivos en los individuos psicóticos. Es decir, las teorías psicológicas sobre juicio de realidad (como p.ej., el modelo de Brickman, 1978; las teorías del reality monitoring de Johnson y Raye, 1981; etc.) aplicadas a la RV podrían llegar a articularse de tal modo que proporcionaran un conjunto de términos relacionados que fueran útiles tanto para describir qué tareas y pruebas utilizar para investigar en este 
campo, como para predecir o definir cuál sería la conducta o ejecución apropiada frente a la que se pudiera comparar la ejecución de un sujeto particular.

Si se probase empíricamente que las personas «normales» siguen los dictámenes de estas teorías, podríamos afirmar que éstas son descriptivamente válidas, al menos en un cierto nivel. Si consideramos las teorías sobre juicio de realidad de que disponemos actualmente, como esquemas normativos, la desviación de cada componente específico, de los que hipotetizan estos modelos (p.ej., la correspondencia interna y la correspondencia externa del modelo de Brickman, etc.), puede que llegue a producir un error en el juicio de realidad diferente en cada caso. En Psicopatología, contar con la referencia de una teoría normativa sobre el juicio de realidad nos ayudaría a identificar cuáles son los déficits concretos de ejecución que cometen las personas que padecen determinados problemas. Pero, incluso si las personas «normales» no se comportaran como las teorías predicen, estas conductas subóptimas (por denominarlas de alguna manera) podrían ofrecernos muchas pistas psicológicas sobre cómo hacen sus juicios de realidad los individuos realmente, preguntándonos si la conducta del individuo se puede describir como una desviación sistemática de la teoría. En este sentido, las teorías sobre juicio de realidad no servirían como esquemas normativos, pero podrían ayudarnos a estudiar empíricamente los errores y sesgos de juicio realidad que se derivasen lógicamente de esas teorías. Por otro lado, y desde un punto de vista práctico, tales desviaciones podrían sugerir intervenciones constructivas que llevaran a un mejor juicio. Es decir, las teorías propuestas por la Psicología podrían ayudar a la Psicopatología y ésta, a su vez, podría ofertar modos de posibles intervenciones que se realizaran desde la Terapia.
Veamos ejemplos concretos de la posible utilidad para la Psicopatología de esa investigación. Por ejemplo, piénsese en la teoría de Brickman (1978), articulada en torno a dos elementos centrales para la atribución de realidad: la correspondencia interna y externa de la conducta. Según Brickman, las personas atribuirán realidad a las experiencias en la medida en que la conducta se corresponda tanto con sentimientos sustanciales y apropiados (correspondencia interna), como con consecuencias importantes (correspondencia externa). Es decir, las situaciones nos parecerán más reales en la medida en que haya emoción en ellas y los errores o aciertos tengan consecuencias importantes.

La correspondencia interna podría tener un papel importante para entender ciertos trastornos. Por ejemplo, en algunos estados disociativos que ocurren en situaciones altamente estresantes y amenazadoras, el individuo puede reaccionar colocando un muro entre sus emociones y su conducta, no permitiendo que ésta se vea alterada por el miedo tan intenso que siente. Según el modelo, esto provocaría una baja correspondencia interna, por lo que la atribución de realidad de esa situación se vería alterada. De hecho, en psicopatología, este tipo de estados se suelen describir como "desapego de la realidad", y el modelo explicaría que este desapego ocurre debido a una baja correspondencia interna junto con una alta correspondencia externa (ya que lo que está en juego en la mayoría de las ocasiones es la propia supervivencia, es decir, la conducta puede tener graves consecuencias), por lo que el individuo se comporta como un autómata, como si no fuera real. De hecho, uno de los objetivos que suele tener la terapia y el consejo psicológico en este tipo de situaciones es provocar explosiones emocionales, es decir, lograr que la situación tenga una correspondencia interna adecuada. Cuan- 
do el individuo revive una y otra vez la situación y logra dar una respuesta emocional, según el modelo, consideraría la situación como más real. Por contra, en las alteraciones fóbicas ocurriría todo lo contrario, el individuo cree que el peligro es real, ya que su conducta está manteniendo una elevada correspondencia interna con su emoción. El individuo siente una elevada ansiedad en la situación, que le lleva a hacer juicios desmesurados de la realidad del peligro. De hecho, creemos que el individuo fóbico realizará con mayor prontitud y confianza atribuciones de realidad en un contexto de RV donde esté presente la situación temida. En uno de los trabajos que estamos realizando actualmente se está intentando evaluar esta hipótesis, comparando las atribuciones de realidad de sujetos normales y de pacientes claustrofóbicos en un ambiente virtual claustrofóbico. Nuestra predicción es que el paciente claustrofóbico creerá con más intensidad que la situación virtual es real. Es decir, su intenso estado emocional en esa situación hará que se sienta «atrapado» por la sensación de realidad, mientras que esto no ocurrirá con tanta intensidad en los sujetos normales, para los que las habitaciones cerradas no serán más que habitaciones virtuales. Para ello, se les pide informes de realidad en diversos momentos, e hipotetizamos que los pacientes claustrofóbicos adscribirán más realidad a medida que el ambiente se vuelva más peligroso para ellos.

Por su parte, la correspondencia externa puede jugar un papel importante en otros trastornos, como por ejemplo, en la depresión. Algunos deprimidos se quejan de cierto desapego de la realidad, de que observan las cosas que ocurren a su alrededor como si se tratase de un gran teatro o de una película de cine. En algunos casos, esto se podría explicar debido a una baja correspondencia externa. Por ejemplo, en la depresión por desesperan- za, planteada por Abramson, Metalsky y Alloy (1989), se postula que el individuo se siente desamparado, sus conductas no tienen consecuencias en el mundo, por mucho que haga no va a cambiar nada. Es decir, según el modelo de Brickman, existiría una baja correspondencia externa y, por tanto, el desamparo debería correlacionar con un sentido de irrealidad de la situación. Por contra, a los pacientes delirantes les ocurriría todo lo contrario. Se podría postular que en una situación de elevada ansiedad, en la que además se considera que las propias conductas tienen consecuencias en el mundo, es más probable que el individuo adscriba realidad a esa situación. Por tanto, si un individuo piensa, por ejemplo, que sus vecinos le acechan y le persiguen (lo que le hace sentir ansiedad) y se esconde para que sus perseguidores no le encuentren, cosa que obviamente ocurre (e.d., no le encuentran, ya que nadie le persigue) atribuirá realidad a esa situación, ya que se corresponde con una elevada correspondencia interna y externa. El problema es que entra en un círculo vicioso, en una profecía auto-cumplida, su conducta recelosa termina provocando conductas similares en los demás, lo que todavía proporciona una mayor correspondencia externa, lo que, a su vez, puede que juegue un papel importante en la confirmación del delirio, en la atribución de realidad de su propia RV.

Las teorías sobre juicio de realidad que surjan a partir de investigaciones en el campo de la RV también podrían ser aplicadas al estudio de las alucinaciones. No existe, hoy por hoy, ninguna teoría que dé una explicación satisfactoria de las alucinaciones. Sin embargo, y a pesar de sus diferencias, todas concuerdan en que éstas representan una ruptura el proceso que permite a las personas distinguir entre acontecimientos mentales privados de acontecimientos públicamente observables (Bentall, 1990). Esta afirmación pre- 
supone que, bajo circunstancias normales, es posible discriminar entre estos dos tipos de acontecimientos. Sin embargo, y como señala Bentall (1990), las teorías actuales sobre alucinaciones suelen olvidarse de que para explicar esto primero deberíamos explicar cómo las personas normales pueden distinguir entre estos dos tipos de acontecimientos. De hecho, resulta tan intrigante el explicar cómo los alucinadores creen que sus alucinaciones son reales, como el explicar cómo las personas normales creemos que los objetos están fuera cuando el procesamiento, que es nuestra experiencia inmediata, ocurre dentro y, sin embargo, otras experiencias, como los sueños, la imaginación o el pensamiento las experimentamos como dentro (Belloch, Baños y Perpiñá, 1995). Si pudiéramos contestar a estas preguntas, las respuestas muy probablemente darían alguna pista de por qué los psicóticos parecen fracasar muy a menudo al hacer esta distinción, ya que se supone que cualquier rasgo o déficit que afecte a la capacidad del individuo para hacer juicios sobre la fuente de su experiencia perceptiva incrementará la probabilidad de que se produzcan alucinaciones (Bentall, 1990). De hecho autores, como Slade, Bentall, Strauss, etc. (p.ej., Bentall, 1990; Launay y Slade, 1981; Strauss, 1969) plantean que existe un continuo entre experiencias alucinatorias y no alucinatorias. El juicio y la discriminación de la realidad presumiblemente sea una habilidad que admita grados en su precisión, como de hecho lo demuestra el que se puedan producir fracasos de estos procesos en individuos normales. Por lo tanto, toda la investigación que en el contexto de RV ayude a conocer cómo y porqué aciertan o fracasan los sujetos en atribuir realidad, son totalmente "transportables» al campo de las alucinaciones. De hecho, y como señala Bentall (1990) es difícil explicar un déficit sin comprender, al menos parcialmente, como ocurre la operación normal.
En primer lugar, la mayoría de teorías afirman que las personas infieren si un acontecimiento es real o imaginario basándose en un amplio rango de información, pero cuando la evidencia disponible es confusa, este tipo de juicios tenderá a ser erróneo. La habilidad para juzgar la fuente de un acontecimiento percibido se suele denominar reality discrimination o discriminación de realidad, y está muy relacionado con el tópico de reality monitoring o supervisión de realidad (Johnson y Raye, 1981). Existen diversas investigaciones sobre las alucinaciones que se enmarcan precisamente dentro de este campo (p.ej., Bentall, Baker y Havers, 1991) y que encuentran que los alucinadores fracasan en aquellas tareas en las que se les pide que discriminen entre recuerdos de acontecimientos internos (e.d., de pensamientos) y recuerdos de acontecimientos externos. En este caso, nos podríamos preguntar qué ocurrirá cuando además de tener huellas de memoria de acontecimientos generados por nosotros mismos y huellas de memoria de acontecimientos reales, también tengamos huellas de memoria de acontecimientos que ocurrieron en RV. ¿Podrán las personas normales discriminar entre estos tres tipos de fuentes? ¿Y los alucinadores, habrá diferencias entre estos tipos de fuentes y la ejecución de normales?

Dejando a un lado estas preguntas tan especulativas, la RV también nos puede ayudar a responder qué tipo de información utiliza el individuo para atribuir un acontecimiento a una fuente externa real y, por tanto, darle juicio de realidad. En un ambiente de RV todos los indicios perceptivos indican que lo que estamos experimentado es real, es más, la persona incluso puede llegar a tener (y, de hecho, lo que se busca es que tenga) la impresión de ser parte de los acontecimientos. Entonces, ¿cómo distingue esa persona qué es real de lo que no lo es? 
(Shapiro y McDonald, 1992). En este contexto seguramente el individuo normal utilizará algún tipo de pista o indicio contextual para atribuir lo que está percibiendo o ha percibido a la RV y no a la realidad física. Es decir, la RV está poniendo de manifiesto que la experiencia y los indicios del pensamiento son más importantes que la riqueza perceptiva. Así, por ejemplo, el recuerdo que tiene la persona de haber entrado en un ambiente de RV puede servir como indicio de que la experiencia no es real. No importa cómo de real sea la experiencia, la mayoría de las personas saben, por ejemplo, que no están realmente volando en un ala delta, que es virtual. Sin embargo, eso no quiere decir que tales experiencias no puedan cambiarnos en distintos modos (Shapiro y McDonald, 1992). En el caso concreto de las alucinaciones, esto nos puede estar indicando que la riqueza perceptiva de la alucinación no es imprescindible para que el individuo crea que lo que está percibiendo no es real, sino que están en juego otras habilidades cognitivas. Y que esos errores pueden tener que ver con el sesgo del alucinador a atribuir los acontecimientos a fuentes externas. Es decir, que las teorías de las imágenes mentales en el campo de las alucinaciones, como p.ej. la de Horowitz (1975), que se basan en la importancia de la viveza y riqueza de los indicios sensoriales a la hora de discriminar la realidad, quedarían, en cierto sentido, en entredicho.

El papel de la información contextual en los fracasos de discriminación de la realidad también se ha subrayado en el trabajo de los psicólogos sociales interesados en la sugestión (Bentall, 1990). Y la sugestionabilidad también ha sido postulada como un factor predisponente a las alucinaciones. En este caso, si se encontraran diferencias individuales en la adscripción de juicio de realidad en contextos de RV en sujetos sugestionables, podríamos reafirmar con mayor seguridad la influencia de este papel en la atribución del juicio de realidad.

Por otro lado, la sugestionabilidad está muy relacionada con las expectativas y, en este sentido, son muchos los autores que plantean que la decisión de si un acontecimiento es real o imaginario está influido por la probabilidad percibida del acontecimiento (Bentall, 1990). Destaca aquí el trabajo de Arieti quien en 1974, y basándose en su trabajo psicoterapéutico con esquizofrénicos, mantenía que éstos sólo oían las voces en ciertas circunstancias, es decir, cuando esperaban oírlas. Eso también es muy probable que ocurra en el contexto de RV. Es decir, cuanto más improbable sea el acontecimiento que el individuo esté experimentando virtualmente, menos atribuciones de realidad hará. Siguiendo con el ejemplo anterior del ala delta, podemos creer que realmente no estamos volando porque recordamos cómo nos pusimos los cascos, o también porque volar en un ala delta es algo que no esperamos que nos pase en el mundo real.

Por otro lado, otra variable que se hipotetiza que puede ser importante a la hora de producir alucinaciones es la ambigüedad estimular. Desde luego, no hace falta insistir en que esta variable se puede manipular de un modo controlado en el ambiente de RV. Uniendo esto al papel de sugestionabilidad, podríamos crear ambientes estimulares ambiguos en RV y generar expectativas de diferentes percepciones mediante instrucciones, para comprobar si estos factores influyen en el juicio de realidad.

Otro factor que se supone que tiene un papel en las alucinaciones es el arousal o activación debida al estrés. Parece, o así lo apuntan Shapiro y McDonald (1992), que bajo ansiedad aguda es más probable que los individuos sean más altamente sugestionables a información que ordinariamente darian por falsa. Es decir, pare- 
ce que la activación, o las emociones intensas en general, tienen un papel importante en la adscripción de realidad. Esto, por otra parte, sería congruente con el modelo de Brickman, donde, como ya hemos visto, se supone que una alta correspondencia interna provocaría más atribuciones de juicio de realidad.

Desde luego, todas las investigaciones que estamos planteando que se pueden realizar en el contexto de la RV, sobre los errores de discriminación de realidad asociados con experiencias alucinatorias, no implican en absoluto que los factores biológicos no sean importantes. En cualquier caso, tampoco creemos que los factores biológicos excluyan a las descripciones y explicaciones que se puedan hacer en términos psicológicos. Si encontramos algún factor de diferencias individuales sistemáticas en la adscripción de juicio de realidad en RV puede que estemos ante un posible factor de vulnerabilidad de padecer alucinaciones. También podríamos especular que si estudiamos los procesos, mecanismos y operaciones implicados en esas confusiones de adscripción de juicio de realidad, podamos estar en un mejor camino para comprender, desde conceptos psicológicos, el fenómeno de las alucinaciones.

Otro tema que, junto a las alucinaciones, es un tópico "estrella» en psicopatología son, sin lugar a dudas, los delirios. Toda teoría que intente aportar comprensión al tema de los delirios debe describir, entre otras cosas, los modos en que el delirante recoge e interpreta la información desde su ambiente social y el modo en que utiliza la evidencia para apoyar o descartar sus creencias (Baños y Belloch, 1995). También debe investigar cómo se forman creencias aberrantes en individuos sanos. De hecho, una estrategia que últimamente está siendo muy fructífera en el estudio de los delirios es el uso de análogos y de creencias manipuladas en el laboratorio, ya que sería realmente interesante demostrar que ciertas condiciones pueden llevar a las personas a volverse recelosas, hostiles y críticas con los demás. En el apartado anterior ya hemos comentado algunas estrategias que desde la RV podrían realizarse para estudiar en este campo. En cualquier caso, no deberíamos pasar por alto que las personas así inducidas no desarrollan necesariamente creencias delirantes. Es decir, existen diferencias importantes entre esas personas, a las que se manipula sus creencias, y los delirantes. Una de esas diferencias es que los sujetos experimentales son conscientes de que están participando en un experimento, que implica en este caso la RV. Y, si es cierto que los delirios son secundarios a experiencias anómalas sin explicación, como afirman teorías tan prestigiosas como las de Maher (1988a y b) estas personas ya tienen una explicación satisfactoria de cualquier experiencia inusual que pudiese ocurrir, y por tanto no necesitan elaborar una explicación delirante.

Por último, la RV no será útil sólo en la investigación sobre el juicio de realidad, sino que también puede ayudar a comprender otros fenómenos psicopatológicos. Por ejemplo, Tart (1990) señala las posibilidades de la RV en el estudio de los estados alterados de conciencia. En cualquier caso, es muy probable que una simulación pueda ser tan compleja como el fenómeno que modela (Korzybski, 1958) pero, aunque sea una abstracción, puede ser una buena herramienta para comprender mejor ese fenómeno. Sin embargo, hay que tener en cuenta la advertencia que hace Tart (1990) de que aunque todos los modelos en ciencia son terriblemente convenientes, también nos pueden llevar peligrosamente por caminos equivocados. Podemos quedarnos fascinados por los modelos, y, aunque el mapa no es el territorio, como muy bien indicaba Korzybski (1958) también hay que añadir que la mayoría de las veces 
preferimos el mapa (Tart, 1990). Por tanto, hay que recordar que los modelos son sólo eso, modelos. Debemos crearlos, usarlos y desecharlos cuando ya empiece a restringirnos.

\section{LA RV COMO ORIGINADORA DE PSICOPATOLOGÍA}

Por último, la RV también puede tener efectos no tan positivos. De hecho, algunos autores (p.ej., Durlach, 1992; Ellis, Kaiser y Grunwald, 1991) mantienen que la $R V$ es un medio que se define en términos de sus efectos sobre los procesos psicológicos tanto básicos como superiores. Y muchos miembros de la comunidad de la RV aceptan el principio de que «el propósito de los computadores es el insight y no los números" (R.Hamming, citado en Brooks, 1988, pág. 7). Esta herramienta, por tanto, puede tener aplicaciones tanto positivas como negativas

En primer lugar, y como señalan Shapiro y McDonald (1992), a medida que los ambientes virtuales se vuelvan parte de nuestros ambientes naturales, se irá desdibujando la distinción entre la realidad del computador y la realidad convencional. Los humanos necesitaremos hacernos más sofisticados en nuestros juicios de realidad, haciendo distinciones entre la realidad física, los artefactos del computador y los artefactos del computador que permiten la manipulación de la realidad física. Desde luego, estas distinciones se volverán cada vez menos importantes a medida que hagamos más y más manipulaciones a través de los artefactos del ordenador. En el caso de la $\mathrm{RV}$ incluso su nombre ya sugiere un posible efecto psicológico que, algunas veces, puede no ser muy deseable: la confusión creciente entre realidad virtual y realidad física. Según Osberg (1997) la RV en vez de una percepción basada en la realidad, se podría definir como una realidad alter- nativa basada en la percepción. Es decir, y como señala Carr (1995) si uno de los aspectos más importantes del pensamiento humano ha sido su capacidad para distinguir entre acontecimientos internos y externos, la RV es el logro de lo opuesto: llevar a las personas a aceptar como real lo que sólo están percibiendo. Si la RV puede afectar al desarrollo cognitivo, para lo bueno y para lo malo, ¿será capaz de llevar a un desarrollo cognitivo distinto del actual? Responder a esta pregunta nos lleva a tomarnos muy en serio a la RV, ya que quizá pueda tener el potencial de remodelar nuestras mentes (Carr, 1995).

Como ya hemos indicado, esta forma de experiencia puede hacer surgir muchas preguntas sobre juicios de realidad, pero incluso también sobre cuestiones morales. Antes ya apuntábamos que, a pesar de que las personas creamos que lo que experimentamos en RV no es real, sin embargo, esto no quiere decir que tales experiencias no puedan cambiarnos (Shapiro y McDonald, 1992). Por ejemplo, ¿se sentirá una persona culpable por cometer adulterio en RV? ¿Cómo se sentirá su esposa? ¿Una persona que haya vivido una guerra virtual podría sufrir un trastorno por estrés post-traumático «virtual»?

Otra de las aplicaciones importantes que tendrá la RV incluye jugar con el concepto de identidad personal (de hecho, esta aplicación ya se está desarrollando). Es decir, la RV puede ser usada tanto para experimentar otra identidad (real o fantástica) como para experimentar otras formas, figuras, objetos e incluso otros modos del self. Según Lanier (Lanier y Biocca, 1992), la definición de cuerpo desde la perspectiva de la RV es aquella parte que puedes mover tan rápido como piensas. Este tipo de definición hace realmente difícil delimitar cuáles son los límites del cuerpo. Desde luego, esta clase de aplicaciones podrá facilitar el aprendizaje de una serie de habilida- 
des cognitivas nuevas. Sin embargo, la RV aplicada así podría también ser especialmente dañina para aquellos que necesitan confrontarse con la realidad y no escapar de ella.

Por otro lado, y salvando las distancias (que son muchas) podría ocurrir algo parecido a lo que ocurre con ciertas drogas, especialmente las psicodélicas. Estas drogas permiten experimentar a los individuos experiencias totalmente nuevas, en las que el self se funde con el mundo $\mathrm{y}$ en las que las percepciones se distorsionan, e incluso se crean percepciones nuevas. La diferencia está, obviamente, en que las drogas actúan directamente sobre nuestro funcionamiento bioquímico. La RV no. Sin embargo, no está claro que la relación sea exclusivamente unidireccional, es decir que las experiencias que tenemos no puedan modelar de algún modo nuestros sistemas orgánicos. Uno de los riesgos de este tipo de drogas es que el uso puede llegar a convertirse en abuso, pero incluso sin una ingesta excesiva y/o desmesurada, el individuo puede llegar a experimentar ciertas experiencias desagradables como los flashbacks, en los que huellas mnésicas de esas experiencias se rememoran de modo no voluntario produciendo, al menos, malestar en quien sufre este fenómeno. ¿Podría producir esto la RV? Eso es algo que todavía no sabemos.

Actualmente, contamos con un cuerpo importante de investigación que examina los efectos que tienen otros medios de comunicación sobre el individuo. Quizás parte de esa investigación se pueda aplicar potencialmente a la RV. Por ejemplo, podemos hipotetizar que, al igual que ha ocurrido con otros medios de comunicación, la RV pueda llegar a generar cierta dependencia. De hecho, parece que los humanos tendemos a sentirnos fascinados por todas aquellas situaciones en las que se supone que confundimos la realidad. Nos fascinan los juegos de magia, las ilusiones perceptivas, etc. Ni qué decir tiene la curiosidad que las drogas alucinógenas despertaron en más de una generación. Esa fascinación inicial a veces se ha convertido en un verdadero problema. Por supuesto, además de la fascinación, en la RV confluyen otros elementos característicos de otros medios gráficos. Baste recordar la dependencia que puede generar la TV, los video-juegos, el ordenador, la comunicación mediante red, etc. Es más que probable que la RV también sea mal usada y abusada por ciertas poblaciones. En cualquier caso, y como ha ocurrido con otras innovaciones y descubrimientos, deberemos aprender a utilizarla.

La investigación sobre otros medios de comunicación (especialmente la TV) también se ha preocupado de los efectos que puede tener la interacción durante tiempo prolongados con ese medio, llegándose incluso a afirmar que el uso continuado de un medio de algún modo sesga la forma en que el individuo piensa y ve el mundo (Biocca, 1992b). Es más, aunque no se sabe con certeza cómo cambian los mecanismos psicológicos de esta persona, lo que sí que se cree es que importa, y mucho, la naturaleza de ese medio, es decir, que los mecanismos de la mente no son indiferentes a los medios por los que se recibe la mayoría de la información. Piénsese que algunos estudios indican que el tiempo medio que una persona emplea mirando la tele es de 7 años de su vida. Si esto mismo se puede aplicar a la $\mathrm{RV}$, esto quiere decir que algunas personas usarán 20 años o más dentro de realidades virtuales (Biocca, 1992b). Incluso directivos de la industria de RV, como Jaron Lanier, proponen que se limite el acceso de los niños a este medio (Lanier y Biocca, 1992). Y es que, lógicamente, esta tecnología podría ser especialmente peligrosa para este tipo de población, del mismo modo que lo puede ser el abuso del vídeo o de los juegos de ordenador o 
de vídeo-consolas. Pero esta nueva herramienta tiene otro riesgo añadido. Los niños necesitan desarrollar muchas habilidades cognitivas para interaccionar con el mundo físico real (p.ej., habilidades viso-manuales, o reflejos motores, etc.). Si el niño utiliza en una edad muy temprana la RV y abusa de ella, estas habilidades pueden no llegar a establecerse totalmente.

Por otro lado, la RV puede ser un escenario idóneo para practicar conductas disfuncionales o antisociales, que podrían incorporarse con facilidad dentro del repertorio conductual. Es decir, la RV también tendrá un impacto importante en nuestra sociedad, al igual que ha ocurrido con otros medios de comunicación. Así, por ejemplo, se han vertido ríos de tinta sobre los peligros potenciales que una mala programación televisiva puede tener sobre los telespectadores, especialmente sobre niños y adolescentes. Algo que se ha visto como especialmente perjudicial es la visión de escenas y películas violentas. Sin embargo, como señalaba Bandura en 1965, se puede aprender el contenido agresivo de la televisión sin que ese conocimiento se traslade a la propia conducta personal. Pero, a diferencia de la televisión, la $\mathrm{RV}$ requiere una acción directa, es decir una persona puede matar o ser matada en esta pseudovida virtual. Como consecuencia, la acción agresiva se puede incorporar directamente dentro del repertorio conductual cuando se juega a juegos de RV o, al menos, así lo demuestran los resultados del estudio de Calvert y Tan de 1994. En este estudio se comparó el impacto de observar escenas violentas versus jugar un juego violento en RV. Se evaluaba la activación fisiológica (mediante la tasa cardíaca), los sentimientos de hostilidad (mediante una versión del Multiple Affective Adjective Check List) y los pensamientos agresivos (mediante un cuestionario de listados de pensamiento). Los resultados mostraron que tanto las medidas fisiológicas como las de pensamientos de agresión eran más elevadas en los sujetos que participaban en el juego, aunque no hubo diferencias en los sentimientos de hostilidad. Es más, los resultados indicaban que las interacciones en RV restaban valor a características, como el género o los rasgos de personalidad hostiles, es decir, la RV producía efectos agresivos similares en todos los jugadores. Por tanto, los autores concluían que la RV es incluso más potente que la diabólica televisión a la hora de suministrar agresión. Aunque la naturaleza simbólica de esta interacción puede sugerir que los participantes no generalizarán estas acciones a la vida real, lo cierto es que la investigación en el campo de la TV sugiere que, desgraciadamente, ocurre lo opuesto (p.ej., Friederich-Cofer y Huston, 1986). Trabajos como los de Schutte, Malouf, Post-Garden y Rodasta (1988) o Silvern y Williamson (1987) indican que las conductas interactivas con vídeo juegos violentos pueden producir también agresión interpersonal. Por tanto, no es extraño que Calvert y Tan se pregunten qué ocurrirá ahora que disponemos de un medio aún más realista: uno en el que la persona puede realmente estar perceptivamente inmersa en un mundo de actividades violentas. Estos autores animan a la investigación en este campo para poder proporcionar guías sobre las potenciales consecuencias conductuales de estas nuevas tecnologías interactivas.

Según las predicciones actuales, los niños que nazcan en los 90 crecerán en un mundo en que la RV será tan disponible y tan barata como pueda ser el teléfono (Lanier y Biocca, 1992). Quizás para entonces el DSM-X o la CIE-14 tenga que incluir algún trastorno relacionado con esta tecnología. Del mismo modo, a lo mejor los manuales terapéuticos incluyan paquetes para utilizarla. En cualquier caso, no queremos cerrar este punto sin 
remarcar que la tecnología no es más que tecnología. La tecnología no hace nada por sí sola.

Para terminar, nos gustaría concluir con la idea con la que hemos iniciado: creemos que la RV se puede constituir en una herramienta que permita a la Psicopatología retar y modificar posiciones teóricas establecidas, a la par que plantearse otras nuevas. Esta nueva forma de experiencia, a la vez que puede ayudarnos a encontrar respuestas a viejas preguntas, puede también hacer surgir otras muchas preguntas nuevas en el horizonte psicopatológico y psicológico. En cualquier caso, y como señala Dennett (1991), es improbable que un ambiente artificial pueda incluso acercarse de lejos a la explosión combinatoria de posibilidades que las acciones humanas pueden realizar.

\section{REFERENCIAS BIBLIOGRÁFICAS}

Abramson, L.Y., Metalsky, G.I., y Alloy, L.Y. (1989). Hopelessness depression: A theorybased subtype of depression. Psychological Review, 96, 358-372.

Aronson, E., y Carlsmith, J.M. (1968). Experimentation in social psychology. En G. Lindzey y E.Aronson (Eds.), Handbook of social psychology (Vol. 2). Reading, Mass.: Addison-Wesley.

Bandura, A. (1965). Influence of models' reinforcement contingencies on the acquisition and performance of imitative responses. Journal of Personality and Social Psychology, 1, 589-595.

Baños, R.M., y Belloch, A. (1995). Psicopatología del pensamiento (II): Los delirios. En A. Belloch, B. Sandín y F. Ramos (Eds.), Manual de Psicopatología (Vol. 1). Madrid: McGraw-Hill.

Begelman, D.A. (1991). Virtual realities and virtual mistakes: A comment on Tart. Dissociation, 4, 214-215.

Belloch, A., Baños, R.M., y Perpiñá, C. (1995). Psicopatología de la percepción y la imginación. En A. Belloch, B. Sandín y F. Ramos (Eds.), Manual de Psicopatología
(Vol. 1). Madrid: McGraw-Hill.

Bentall, R.P. (1990). The illusion of reality: A review and integration of psychological research on hallucinations. Psychological Bulletin, 107, 82-95.

Bentall, R.P., Baker, G.A., y Havers, S. (1991). Reality monitoring and psychotic hallucinations. British Journal of Clinical Psychology, 30, 213-222.

Biocca, F. (1992a). The problem of simulation sickness and the diffusion of virtual reality techology. Trabajo presentado en International Communication Association, Miami.

Biocca, F. (1992b). Communication within virtual reality: Creating a space for research. Journal of Communication, 42, 5-22.

Brickman, P. (1978). Is it real? En H. Harvey, W. Ickes y R.F. Kidd (Eds.), New directions in attribution research (Vol. 2). Hillsdale: Erlbaum.

Brooks, F. (1988). Grasping reality through illusion: Interactive graphics serving science. Chape-Hill: Department of Computer Science. University of North Carolina at Chapel Hill.

Calvert, S.L., y Tan, S.L. (1994). Impact of virtual reality on young adult's psysiological arousal and aggressive thougts: Interaction versus observation. Journal of Applied Developmental Psychology, 15, 125-139.

Carr, K. (1995). Introduction. En K. Carr y R. England (Eds.), Simulated and virtual realities. Londres: Taylor \& Francis.

Dennett, D.C. (1991). Conscioussness explained. Boston: Little, Brown and Co.

Durlach, N. (1992). Virtual environment techonology for training. Cambridge, MA: Virtual Environment and Teleoperator Research Consortium, MIT-BBN Systems and Technologies.

Ellis, S.R., Kaiser, M., y Grunwald, A. (1991). Pictorial communication in virtual and real evironments. Londres: Taylor \& Francis.

Friedrich-Cofer, L., y Huston, A.H. (1986). Television violence and aggression: The debate continues. Psychological Bulletin, 100, 364-371.

Horowitz, M. (1975). A cognitive model of hallucinations. American Journal of Psychiatry, 132, 789-795. 
Johnson, M.K., y Raye, C.L. (1981). Reality monitoring. Psychological Review, 88, 6785.

Korzybski, A. (1958). Science and sanity: An introduction to non-aristotelian systems and general semantics. Lakeville, Connecticut: The International Non-Aristotelian Publishing Co.

Lanier, J., y Bicoa, F. (1992). An insider's view of the future of Virtual Reality. Journal of Communication, 42, 150-172

Launay, G., y Slade, P.D. (1981). The measurement of hallucinatory predisposition in male and female prisoners. Personality and Individual Differences, 2, 221-234.

Maher, B.A. (1988a). Anomalous experience and delusional thinking: The logic of explanations. En T.F. Oltmanns y B.A. Maher (Eds.), Delusional Beliefs. N.Y.: Wiley \& Sons.

Maher, B.A.(1988b). Delusions as the product of normal cognitions. En T.F. Oltmanns y B.A. Maher (Eds.), Delusional Beliefs. N.Y.: Wiley \& Sons.

Maher, B.A., y Spitzer, M. (1992). Delusions. En P.B. Sutker y H.E. Adams (Eds.), Comprehensive Handbook of Psychopathology ( $2^{\mathrm{a}}$ ed.). N.Y.: Plenum Press.

Perpiñá, C., Botella, C., y Baños, R.M. (1997). Realidad Virtual y Psicología: Un heurísti- co en ambas direcciones. Psicologemas, 21, 127-146.

Osberg, K. (1997). But what's behind door number 4???. Seattle, WA: Human Interface Technology Laboratory.

Schutte, N., Malouf, J., Post-Garden, J., y Rodasta, A. (1988). Effects of playing videogames on children's aggessive and other behaviors. Journal of Applied Social Psychology, 18, 454-460.

Shapiro, M.A., y McDonald, D.G. (1992). I'm not a real doctor, but I play one in Virtual Reality: Implications of Virtual Reality for judgments about reality. Journal of Communication, 42, 94-114.

Silvern, S.B., y Williamson, P.A. (1987). The effects of video game play on young children's aggession. Journal of Applied Developmental Psychology, 8, 453-462.

Strauss, J.S. (1969). Hallucinations and delusions as points of continual function. Archives of General Psychiatry, 21, 581586.

Tart, C.T. (1990). Multiple personality, altered states and virtual reality: The world simulation process approach. Dissociation, 3, 222-233.

Tart, C.T. (1991). On the use of computergenerates realities: A Response to Begelman. Dissociation, 4, 216-217. 